\title{
Development of a Novel Bulk Sample TEM Heater and Its Application
}

\author{
Meng $\mathrm{Li}^{{ }^{1}}$, De-Gang $\mathrm{Xie}^{1}$ and $\underline{\mathrm{Zhi}-\text { Wei Shan }}{ }^{1}$ \\ 1. Center for Advancing Materials Performance from the Nanoscale (CAMP-Nano), State Key \\ Laboratory for Mechanical Behavior of Materials, Xi'an Jiaotong University, Xi'an, China \\ ${ }^{\dagger}$ Present address: Department of Chemical and Petroleum Engineering, University of Pittsburgh, \\ Pittsburgh, PA, USA
}

Understanding the microstructure evolution of bulk materials such as metals and alloys under different temperatures is critical for improving the design, processing, performance and safety of these materials. In recent years, it has become a trend to use MEMS based technologies for the development of in-situ TEM heating devices, due to the greatly improved image stability and dramatically reduced drift settle time. However, current MEMS heaters are mainly designed for applications in nano-structured materials instead of bulk samples. Unlike nano-structured materials such as nanoparticles and nanowires which are electron transparent and therefore can be observed directly inside TEM, bulk samples need to experience a series of thinning procedure (e.g. electropolishing, FIB milling and/or ion milling) before they become thin enough for TEM observations. Current MEMS membrane heaters are usually very arduous for bulk sample transfer. In addition, during temperature change, the heating membrane suffers from unavoidable bulging problem, which in turn changes the height of the sample inside the TEM. Consequently, it is very difficult to record successive microstructural evolution clearly, especially at atomic resolution.

In this work, we develop a novel MEMS heater for bulk sample that facilitates bulk sample preparation and is capable of atomic scale imaging even with ramping temperature. The heater consists of a cantilevered hotplate with sample mounting bars for FIB. The hotplate is isolated with the rest part of the chip by springs as shown in Figure. 1 (a). Our design has several advantages: first of all, bulk sample can be easily prepared onto this heater using standard FIB liftout process; second, this design enables further thinning, fabricating or even nanomilling on the FIB transferred sample to reduce FIB damage and get high quality TEM sample; thirdly, the springs connecting the freestanding hotplate and the rest part of the chip can counterbalance the thermal expansion of the hotplate, which in turn solves the bulging problem.

Using this special home-made ultra-stable in-situ heating system and the state-of-art environmental TEM(ETEM), hydrogen induced interface failure on aluminum was studied [1]. Hydrogenated aluminum nanopillars [2] fabricated from bulk pure aluminum was heated up inside an ETEM. The interface evolution throughout the heating process was observed from the edge-on view. We found that hydrogen exposure for just a few minutes can greatly sabotage the high-temperature integrity of the metal-oxide interface. Even for samples without obvious surface blistering, giant cavities can form at the metal side at elevated temperatures, while the surface oxide layer remains unchanged. Such giant cavities can become hidden threat for the safety of the material due to intact oxide layer. Moreover, a critical temperature of $\sim 150 \mathrm{C}$ was found, above which the growth of interfacial nano-cavities reversed to shrink, followed by the formation of a few giant cavities. Based on the collected data, we propose that vacancy supersaturation, activation of long-range 
diffusion pathway along the detached interface and dissociation of hydrogen-vacancy complexes are the critical factors for this behavior. These results are expected to shed new light on understanding of hydrogen-induced interfacial failures at elevated temperature [3].

\section{References:}

[1] M. Li et al, Nature Communications, 10.1038/NCOMMS14564 (2017).

[2] D. Xie, et al, Nat. Mater., 14 (2015), p. 899.

[3] The authors acknowledge the supports by the National Key Research and Development Program of China (No. 2017YFB0702001), Natural Science Foundation of China (51621063) as well as the support from Prof. Xixiang Zhang in King Abdullah University of Science and Technology (KAUST) for MEMS fabrication.
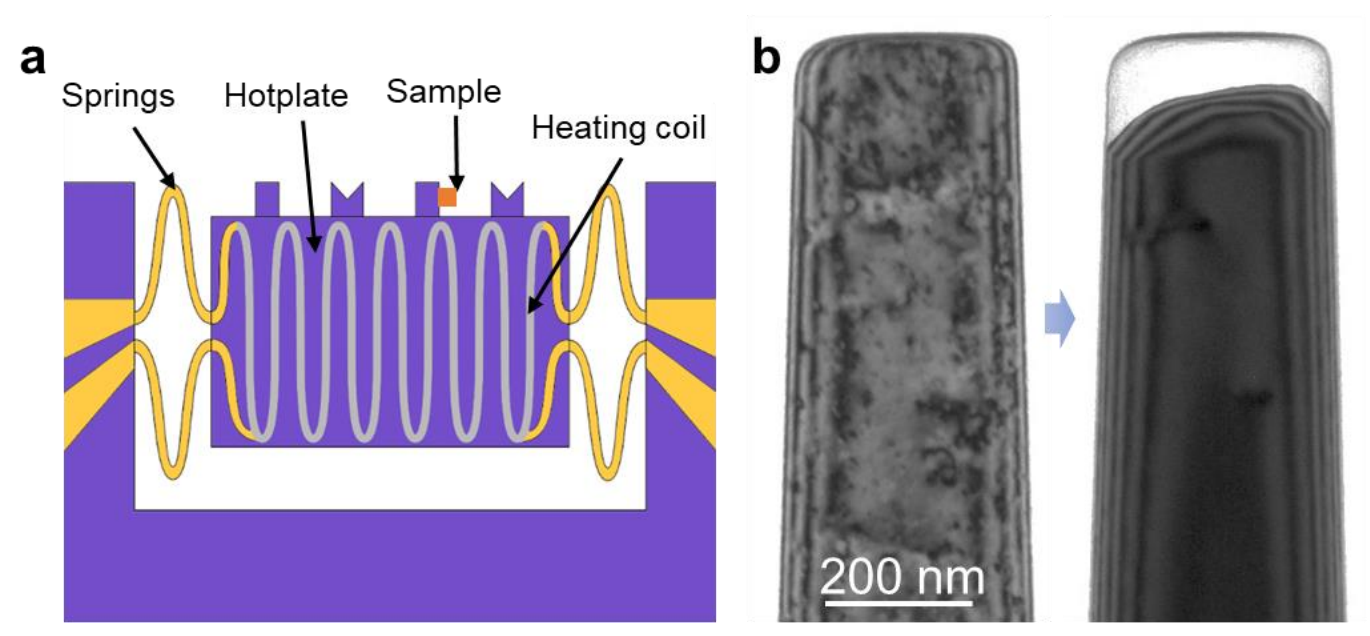

Figure 1. (a)Schematic illustration of the home-made MEMS heating chip with precise temperature sensing and ultra-high image stability.

(b) TEM bright field image of the hydrogenated pillar before(left) and after(right) heating. Note the giant cavity at the top of pillar on the metal side. 